BMJ Open

Diabetes

Research

\& Care

\title{
Effects of carbohydrate quality and amount on plasma lactate: results from the OmniCarb trial
}

\author{
Jiun-Ruey Hu (D , ${ }^{1}$ Yingfei Wu, ${ }^{2}$ Frank M Sacks, ${ }^{3}$ Lawrence J Appel, ${ }^{2}$ \\ Edgar R Miller III, ${ }^{2}$ J Hunter Young, ${ }^{2}$ Stephen P Juraschek (D) ${ }^{4}$
}

\begin{abstract}
To cite: Hu J-R, Wu Y, Sacks FM, et al. Effects of carbohydrate quality and amount on plasma lactate: results from the 0mniCarb trial. BMJ Open Diab Res Care 2020;8:e001457. doi:10.1136/ bmjdrc-2020-001457
\end{abstract}

- Additional material is published online only. To view please visit the journal online (http://dx.doi.org/10.1136/ bmjdrc-2020-001457).

This project was presented as a poster at the 2019 Scientific Sessions of the American Heart Association (AHA) in Philadelphia, Pennsylvania on November 16, 2019.

Received 14 April 2020 Revised 16 July 2020 Accepted 21 July 2020

Check for updates

(C) Author(s) (or their employer(s)) 2020. Re-use permitted under CC BY-NC. No commercial re-use. See rights and permissions. Published by BMJ.

For numbered affiliations see end of article.

Correspondence to Dr Stephen P Juraschek; sjurasch@bidmc.harvard.edu

\section{ABSTRACT}

Introduction Plasma lactate is a marker of non-oxidative glucose metabolism associated with progression to diabetes. We examined the effect of carbohydrate quality (glycemic index (Gl)) and amount (\%kcal) on plasma lactate. We hypothesized that low $\mathrm{Gl}(\leq 45 \mathrm{~g})$ ) versus high $(\geq 65(\mathrm{G}))$ and low \%kcal from carbohydrate $(40 \% \mathrm{kcal}$ (c)) versus high $(58 \% \mathrm{kcal}(\mathrm{C}))$ each would reduce lactate levels.

Research design and methods We measured lactate in OmniCarb, a randomized, cross-over trial of four diets in overweight/obese adults without diabetes or cardiovascular disease $(\mathrm{N}=163)$. The four diets were high carbohydrate+high Gl (CG, reference), high carbohydrate+low Gl $(\mathrm{Cg})$, low carbohydrate+high Gl (cG), and low carbohydrate+low Gl (cg). Participants ( $\mathrm{N}=163)$ consumed each of the four diets over a 5-week period, separated by 2 -week washout periods. Plasma lactate levels were measured at baseline, during which the participants consumed their own diets, and after each 5-week period.

Results Baseline plasma lactate was $1.2 \mathrm{mmol} / \mathrm{L}$. In the setting of high carbohydrate amount, reducing Gl lowered plasma lactate non-significantly by $0.08 \mathrm{mmol} / \mathrm{L}$ (Cg vs CG: $95 \% \mathrm{Cl}-0.16$ to $0.00 ; \mathrm{p}=0.06)$. In the setting of high $\mathrm{Gl}$, reducing carbohydrate amount lowered plasma lactate by $0.10 \mathrm{mmol} / \mathrm{L}$ (cG vs CG: $95 \% \mathrm{Cl}-0.19$ to $-0.02 ; \mathrm{p}=0.02$ ). The combined effect of reducing $\mathrm{Gl}$ and carbohydrate proportion in the diet (cg vs CG) was similar (cg vs CG: $-0.08 ; 95 \% \mathrm{Cl}-0.16$ to $0.00 ; \mathrm{p}=0.04$ ). All four diets reduced plasma lactate compared with baseline. Conclusions Compared with a diet with high Gl and high carbohydrate amount, diets with low Gl and/or low carbohydrate amount reduced plasma lactate. Whether this change in lactate leads to long-term change in glucose metabolism needs to be examined.

Trial registration number NCT00608049.

\section{INTRODUCTION}

Diabetes mellitus is a major and growing public health problem, with an annual incidence of 1.5 million in the USA. ${ }^{1}$ There is a critical need for population-wide strategies to address this epidemic. Among the many dietary determinants of diabetes, carbohydrates have the most potent effects on glucose and insulin secretion. Two features

\section{Significance of this study}

What is already known about this subject?

- Carbohydrate glycemic index (Gl) and amount have been shown to worsen glucose control among adults with diabetes, but whether these two features of carbohydrates impact the risk of developing diabetes in at-risk adults is unknown and intensely debated.

What are the new findings?

- Compared with a diet with high $\mathrm{Gl}$ and high carbohydrate amount, diets with low Gl and/or low carbohydrate amount reduced plasma lactate.

How might these results change the focus of research or clinical practice?

- Whether this change in lactate reduces long-term diabetes risk should be examined.

of dietary carbohydrates, quality (glycemic index (GI), ie, how fast specific foods raise blood glucose in the 2 hours after consumption) and amount (proportion of calories), have been shown to worsen glucose control among adults with diabetes, with higher GI and higher carbohydrate amount causing poorer glucose control. ${ }^{2-4}$ However, whether these two features of carbohydrates impact the risk of developing diabetes among adults at elevated risk is unknown and is intensely debated. $^{56}$

There have been significant challenges to defining causal pathways between consumed carbohydrates and diabetes. Randomized controlled feeding studies of carbohydrate quality and amount have been too short to observe diabetes as an outcome. One such trial, the Effect of Amount and Type of Dietary Carbohydrates on Risk of Cardiovascular Heart Disease and Diabetes Study (OmniCarb), examined the impact of modifying carbohydrate GI and amount over 5 weeks on cardiovascular risk factors among relatively healthy adults who were overweight or obese. $^{7}$ This trial found that neither varying 
carbohydrate amount nor GI improved insulin sensitivity or intermediate-term markers of glucose homeostasis, ${ }^{7-9}$ leading some to question the role of GI, carbohydrate amount, and dietary carbohydrates in general in the development of diabetes. ${ }^{6}$ However, because OmniCarb was conducted in people with intact regulatory systems for blood glucose, blood glucose may not have been sensitive enough to detect short-term dietary effects on glucose homeostasis. This raises the question of whether other subclinical markers would be more appropriate for observing short-term changes in metabolism that might represent progression toward diabetes.

In recent years, plasma lactate has emerged as an early marker of abnormal metabolism that is strongly associated with insulin resistance and the development of diabetes mellitus in large human cohort studies. ${ }^{10-13}$ In adults without diabetes, pyruvate, the main product of intracellular glucose metabolism, is converted to lactate in nonoxidative glycolysis. ${ }^{14}$ In persons with insulin resistance, this process is enhanced, ${ }^{15}$ contributing to elevations in lactate well before impairments in glucose regulation are observed. ${ }^{16-18}$ Furthermore, a number of physiologic studies suggest that elevated lactate might even accelerate diabetes progression by promoting gluconeogenesis and reducing muscle reuptake of glucose ${ }^{19-21}$ As a result, lactate may be a useful early marker of diabetes pathogenesis with causal implications.

The objective of the present study was to determine the effect of diets that vary in GI and carbohydrate amount on plasma lactate in overweight and obese adults without diabetes. We hypothesized that lowering the GI and lowering the amount of carbohydrates would alter intracellular metabolism and thereby reduce plasma lactate levels. In addition, we compared lactate with traditional diabetes risk factors and markers of glycemia or insulin resistance.

\section{RESEARCH DESIGN AND METHODS}

Trial design

OmniCarb was an investigator-initiated, National Heart, Lung, and Blood Institute-supported trial whose rationale and main results have been previously published. ${ }^{7}$ In brief, OmniCarb was a randomized, controlled, cross-over trial in which participants consumed four distinct diets in random order over four dietary intervention periods. The diets varied by GI $(\geq 65$ in the high GI group, $\leq 45$ in the low GI group) and by carbohydrate amount $(40 \% \mathrm{kcal}$ in the low carbohydrate group, $58 \% \mathrm{kcal}$ in the high carbohydrate group), to create four distinct diets: high carbohydrate and high GI (CG), high carbohydrate and low GI (Cg), low carbohydrate and high GI (cG), and low carbohydrate and low GI (cg). A detailed nutrient breakdown of the four diets is presented in online supplementary table $\mathrm{S} 1$. GI is a measure of the degree of blood glucose rise after a standardized amount of carbohydrate intake, based on the area under the glucose curve during the post-carbohydrate 2-hour period, relative to a standard dose of $100 \mathrm{~g}$ of white bread. ${ }^{4}$ The cut points in glycemic index (GI < 45 vs GI >65) correspond to the first and fifth quintiles of US population-based intake. $^{22}$ There were eight possible sequences of the four intervention diets. ${ }^{7}$ Diet sequences were stratified by site to ensure a balance of sequences at each site in each cohort. Randomly varying block sizes were used to prevent predictability of sequence assignment. Kitchen staff needed to have knowledge of participant diet assignment. All lab personnel were blinded to diet sequence assignment. Until the end of the trial, all investigators, staff and participants were masked to all trial outcome data, with the exception of the trial statisticians, the data manager, and the External Monitoring Committee. Modifications to the trial protocol, including the complete protocol, have been published previously. $^{7}$

\section{Participant recruitment}

Participants of the trial were obese or overweight adult men and women enrolled at two clinical centers, one located in Boston, Massachusetts, and the other in Baltimore, Maryland, USA. Participants were excluded if they reported a prior diagnosis of diabetes mellitus or cardiovascular disease, if they had a fasting blood glucose $>125 \mathrm{mg} / \mathrm{dL}$, or if they were taking medications for blood pressure, lipids, or diabetes (online supplementary table S2). All participants provided informed consent before taking part. The participant flow diagram is shown in figure 1.

\section{Controlled feeding}

Feeding was conducted by cohort, between August 2009 and September 2010. Participants were randomly assigned to one of eight sequences of four diet periods. All participants began with an 8-day run-in phase in which each of the four study diets was given for 2 days. Participants then consumed each diet over a 5 -week period, followed by a 2-week washout, during which they ate a self-selected diet. The intervention was designed to last 5 weeks based on findings from previous studies of carbohydrates and GI that found significant effects on insulin sensitivity in 2-4 weeks. ${ }^{7}$ Attendance at meals was recorded. Participants were required to eat one principal meal on-site each day and were observed while eating. Participants were sent home with the remaining two meals and weekend meals. A complete list of sample food items organized by diet can be found in online supplementary tables S3-S23. Every day, participants completed a diary in which they listed their consumption of protocol and non-protocol foods. Adherence was high; $96 \%$ of person-days consisted of study food consumption without consumption of nonstudy foods. Any alcohol consumption was reported on $11 \%$ of person-days.

Each diet was designed to be healthful, with core features of the Dietary Approaches to Stop Hypertension diet, that is, reduced in saturated fat, sodium, and cholesterol, while rich in fruits, vegetables, fiber, potassium, 


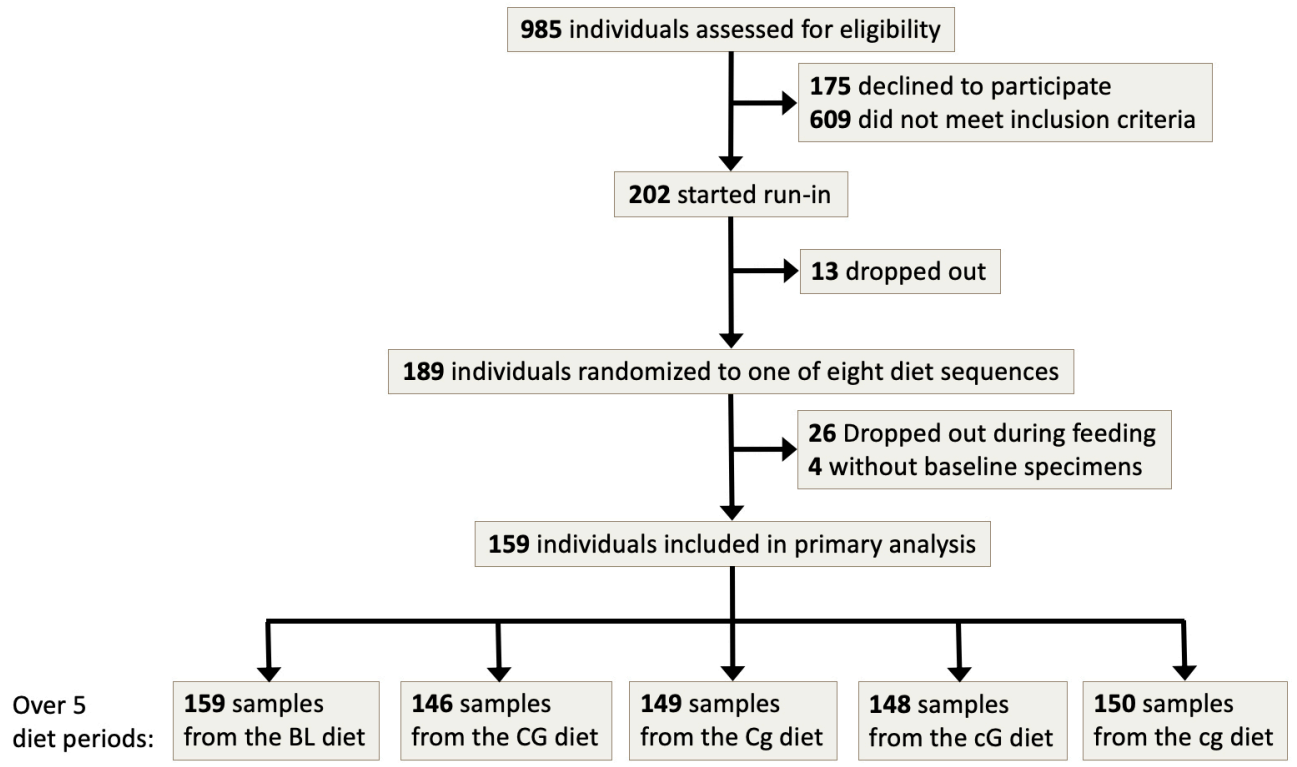

Figure 1 Participant flow diagram in the OmniCarb study. BL, baseline; CG, high carbohydrate, high glycemic index diet; $\mathrm{Cg}$, high carbohydrate, low glycemic index diet; cG, low carbohydrate, high glycemic index diet; cg, low carbohydrate, low glycemic index diet.

and other minerals at recommended levels. Sodium was similar in all four diets. Calorie targets were determined for each participant based on their body size, sex, and physical activity level. Calorie intake was adjusted throughout the trial to keep weight within $2 \%$ of participants' baseline values. Participants were encouraged to maintain the same activity levels and alcohol consumption throughout the study. Since many low GI foods are high in fiber and many high GI foods are low in fiber, similar fiber contents were established across diets by including low fiber, low GI foods such as pasta, and high fiber, high GI foods such as instant oatmeal and bananas. This issue was also addressed by adding unprocessed wheat bran to three of the breakfasts and two to three dinners per week. At the $2000 \mathrm{kcal}$ level, dietary fiber was targeted to a range of 26-31 $\mathrm{g}$ a day, and ultimately ranged from $29 \mathrm{~g}$ to $37 \mathrm{~g}$.

\section{Measurement of outcomes}

Fasting plasma samples were collected at baseline prior to randomization and at the completion of each 5-week fasting period. The mean number of weeks between laboratory measurements in consecutive diet periods was 7.6 (SD: 2.0). Glucose and insulin levels were measured in plasma after a brief storage period soon after specimen collection as part of the original trial protocol. ${ }^{7}$ Additional plasma specimens were stored at $-70^{\circ} \mathrm{C}$ until a single thaw cycle in 2012 to measure lactate. Lactate was measured via a lactate-pyruvate oxidation reaction by a Dimension Vista analyzer (Siemens). The observed interassay coefficient of variation for lactate was 2.1\% (corresponding to a mean of $3.325 \mathrm{mmol} / \mathrm{L}$ ). In a general healthy population without diabetes, plasma lactate should be $0.5-1 \mathrm{mmol} / \mathrm{L}$.

\section{Other covariate measurements and definitions}

In addition to the primary outcome of our study, covariates were ascertained via questionnaire, laboratory measurement, and physical examination. The Food Frequency Questionnaire was inputted by participants directly into the web-based system of the National Cancer Institute. ${ }^{23}$ Body mass index (BMI) was calculated using baseline height and weight and categorized as overweight $\left(25-29.9 \mathrm{~kg} / \mathrm{m}^{2}\right)$ or obese $(\geq 30 \mathrm{~kg} /$ $\mathrm{m}^{2}$ ). Weight was measured with a calibrated Tanita BWB-800 digital scale. Height was measured with a stadiometer. Waist circumference $(\mathrm{cm})$ was measured with an anthropometric measuring tape, $1 \mathrm{~cm}$ above the navel. Impaired fasting glucose was based on a fasting blood glucose $\geq 100 \mathrm{mg} / \mathrm{dL}$. The homeostasis model assessment (HOMA) index was calculated by multiplying fasting serum insulin level $(\mu \mathrm{U} / \mathrm{mL})$ and fasting serum glucose concentration $(\mathrm{mg} / \mathrm{dL})$ divided by $405 .{ }^{24}$ Ultracentrifugation was used to prepare lowdensity lipoprotein for cholesterol measurements. High-density lipoprotein-cholesterol was measured by a precipitation method using dextran sulfate and magnesium chloride. Cholesterol and triglycerides were measured by enzymatic assays. Hypertensive status (yes or no) was determined by an average of three baseline blood pressure measurements for which mean systolic blood pressure was $\geq 140 \mathrm{~mm} \mathrm{Hg}$ or mean diastolic blood pressure was $\geq 90 \mathrm{~mm} \mathrm{Hg}$ (consistent with the definition of hypertension at the time the study was conducted).

\section{Statistics}

The main outcomes were (1) between-diet differences in end-of-period plasma lactate levels and (2) change 
in plasma lactate from baseline in each of the four diets. Given the trial design, the primary contrasts of interest were the between-diet differences in the endof-period levels of plasma lactate. Accounting for the repeated measures design, all comparisons were done using generalized estimating equation linear regression models, with a Huber and White robust variance estimator which assumed an exchangeable working correlation matrix. In addition, we examined the cross-sectional association between baseline lactate and markers of glycemia and insulin resistance using Pearson's coefficients and linear regressions. We also used generalized estimating equations adjusted for sex (female, male), age (continuous), and race/ethnicity (non-Hispanic African-American, non-Hispanic white, Hispanic, non-Hispanic Asian) to examine the crosssectional association between markers using repeat measurements. Lastly, we performed stratified analyses by age (below or above 65 years), sex, race, obesity status, and HOMA-Insulin Resistance (IR) (below or above median). In the stratified analyses, $p$ values for each stratum were generated using interaction terms. All analyses were performed in Stata V.15.1. Statistical significance was defined as $\mathrm{p} \leq 0.05$ without Bonferroni correction.

\section{RESULTS}

\section{Baseline characteristics}

Baseline characteristics of this randomized study population are shown in table 1 . The 163 participants had a mean age of 53 years (SD: 11); $52 \%$ of the participants were women, $40 \%$ were non-Hispanic AfricanAmerican, $56 \%$ were obese and $26 \%$ had hypertension. Participants had a baseline lactate level of $1.2 \mathrm{mmol} / \mathrm{L}$ (SD: 0.5).

\section{Change in plasma lactate from baseline by diet}

All four diets reduced lactate compared with baseline, with magnitudes ranging from -0.10 to $-0.20 \mathrm{mmol} / \mathrm{L}$ (table 2).

\section{Between-diet comparisons}

We first compared one diet factor (GI or \% kcal carbohydrate) while holding the other factor constant (figure 2). In the setting of high carbohydrate amount, reducing GI lowered plasma lactate by $0.08 \mathrm{mmol} / \mathrm{L}$ (Cg vs CG: $95 \%$ CI -0.16 to $0.00 ; p=0.06)$. In the setting of low carbohydrate amount, reducing GI did not reduce plasma lactate (cg vs cG: mean change of $0.02 \mathrm{mmol} / \mathrm{L} ; 95 \% \mathrm{CI}-0.06$ to $0.10 ; \mathrm{p}=0.60)$. In the setting of high GI, reducing carbohydrate amount lowered plasma lactate by $0.10 \mathrm{mmol} / \mathrm{L}$ (cG vs CG: $95 \%$ CI -0.19 to $-0.02 ; p=0.02$ ). In the setting of low GI, reducing carbohydrate amount did not reduce plasma lactate (cg vs $\mathrm{Cg}$ : mean change of $0.00 \mathrm{mmol} / \mathrm{L}$; $95 \%$ CI -0.08 to $0.07 ; \mathrm{p}=0.90)$.

We also compared diets differing in both variables. The combined effect of reducing both GI and carbohydrate proportion (CG vs $\mathrm{cg}$ ) was similar to reducing
Table 1 Baseline characteristics of participants $(N=163)$ in the OmniCarb trial

\begin{tabular}{lc}
\hline Characteristics & $\begin{array}{c}\text { Mean (SD) or } \\
\mathbf{n}(\%)\end{array}$ \\
\hline Age, years & $52.6(11.4)$ \\
\hline Women, \% & $85(52)$ \\
\hline Race, \% & \\
\hline Non-Hispanic African-American & $66(40)$ \\
\hline Non-Hispanic white & $82(50)$ \\
\hline Hispanic & $11(7)$ \\
\hline Asian & $4(2)$ \\
\hline Lactate, mmol/L & $1.2(0.5)$ \\
\hline Body mass index, kg/m ${ }^{2}$ & $32.3(5.5)$ \\
\hline Body mass index, \% & \\
\hline 25-29.9 & $71(44)$ \\
\hline$\geq 30$ & $92(56)$ \\
\hline Waist circumference, $\mathrm{cm}$ & $104.4(13.5)$ \\
\hline Serum fasting glucose, $\mathrm{mg} / \mathrm{dL}$ & $97.3(13.6)$ \\
\hline Serum 2-hour glucose, $\mathrm{mg} / \mathrm{dL}$ & $135.4(42.6)$ \\
\hline Serum fasting insulin, $\mu \mathrm{U} / \mathrm{mL}$ & $7.7(5.8)$ \\
\hline Serum 2-hour insulin, $\mu \mathrm{U} / \mathrm{mL}$ & $48.8(43.1)$ \\
\hline Impaired fasting glucose (serum fasting & $58(35.6)$ \\
glucose $\geq 100 \mathrm{mg} / \mathrm{dL}), \%$ & $1.9(1.6)$ \\
\hline Homeostasis model assessment, units & $58.3(16.0)$ \\
\hline HDL cholesterol, mg/dL & $153.0(42.1)$ \\
\hline LDL cholesterol, mg/dL & $104.6(67.1)$ \\
\hline Triglycerides, mg/dL & $132.0(9.1)$ \\
\hline SBP, mm Hg & $80.0(7.5)$ \\
\hline DBP, mm Hg & $120(74)$ \\
\hline Baseline hypertensive status ${ }^{\star}, \%$ & $43(26)$ \\
\hline Non-hypertensive & \\
\hline Hypertensive & \\
\hline
\end{tabular}

*Defined as baseline SBP $\geq 140$ or DBP $\geq 90 \mathrm{~mm} \mathrm{Hg}$.

DBP, diastolic blood pressure; HDL, high-density lipoprotein; LDL, low-density lipoprotein; SBP, systolic blood pressure.

either, with a mean change of $-0.08 \mathrm{mmol} / \mathrm{L}(95 \% \mathrm{CI}$ -0.16 to $0.00 ; p=0.04)$. There was no significant difference in lactate change between the high carbohydrate, low GI diet and the low carbohydrate, high GI diet, with a mean change of $-0.03 \mathrm{mmol} / \mathrm{L}$ ( $\mathrm{Cg}$ vs cG: $95 \%$ CI -0.10 to $0.05 ; \mathrm{p}=0.51$ ).

\section{Lactate, markers of glycemia, and risk factors for hyperglycemia}

We examined the relationship between lactate and markers of glycemia and insulin resistance. Baseline lactate levels were positively correlated with markers of glycemia and insulin resistance, including serum fasting glucose, serum 2-hour glucose, serum fasting insulin, serum 2-hour insulin, and HOMA (table 3). After adjustment for age, sex, and race/ethnicity, significant positive 
Table 2 Change in plasma lactate level compared with baseline $(n=159)$

\begin{tabular}{llr}
\hline Diet & $\boldsymbol{\beta}(\mathbf{9 5 \%} \mathbf{C l})$ & P value \\
\hline $\mathrm{CG}$ & $-0.10(-0.18$ to -0.02$)$ & 0.012 \\
$\mathrm{Cg}$ & $-0.18(-0.27$ to -0.09$)$ & $<0.001$ \\
$\mathrm{CG}$ & $-0.20(-0.29$ to -0.11$)$ & $<0.001$ \\
$\mathrm{Cg}$ & $-0.18(-0.26$ to -0.11$)$ & $<0.001$ \\
\hline
\end{tabular}

Participants had a baseline lactate level of $1.2 \mathrm{mmol} / \mathrm{L}$ (SD: 0.5$)$.

$P$ value refers to the $p$ value for linear regression based on $a$ repeated measures analysis.

$\beta$, beta coefficient; $C G$, high carbohydrate, high glycemic index diet; cG, low carbohydrate, high glycemic index diet; $\mathrm{Cg}$, high carbohydrate, low glycemic index diet; cg, low carbohydrate, low glycemic index diet.

associations with lactate were demonstrated with serum fasting glucose, serum 2-hour glucose, serum fasting insulin, serum 2-hour insulin, and HOMA.

\section{Sensitivity analyses}

In subgroup analyses, the between-diet change in lactate did not differ by age, sex, race, BMI, hypertension status, baseline fasting glucose, and HOMA score (online supplementary table S24).

\section{DISCUSSION}

In this randomized, controlled feeding study that enrolled persons without diabetes, diets with low GI and/or low carbohydrate amount reduced plasma lactate compared with diets with high GI and high carbohydrate amount. The combined effect on lactate of reducing GI and carbohydrate proportion in the diet was similar to the individual reductions in GI or carbohydrate, a

\section{Between Diet Comparisons of Change in Lactate}

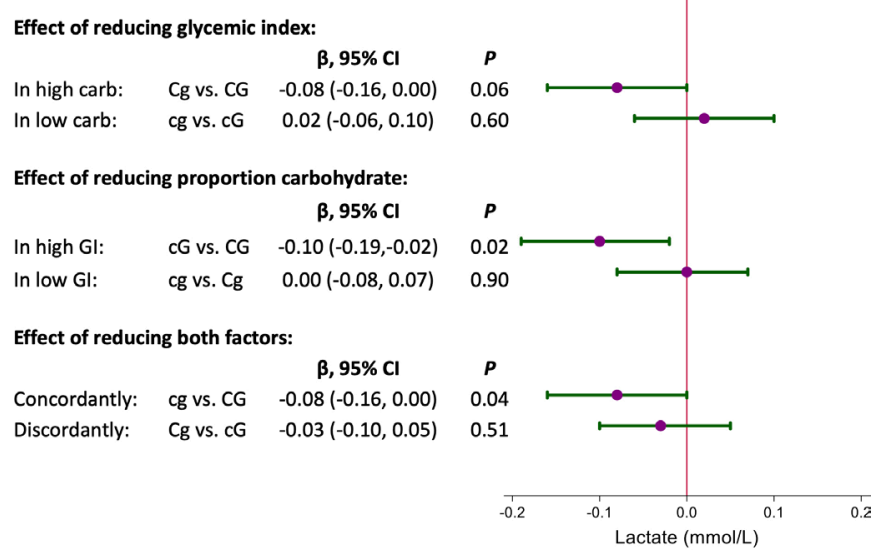

Figure 2 Between-diet comparisons of change in lactate. In each comparison, the result displayed is the first diet compared with the second diet. For example, cg vs cG means cg minus cG, and so on. CG, high carbohydrate, high glycemic index diet; $\mathrm{Cg}$, high carbohydrate, low glycemic index diet; cG, low carbohydrate, high glycemic index diet; cg, low carbohydrate, low glycemic index diet; Gl, glycemic index.
Table 3 Linear associations and Pearson's correlation coefficients between markers of glycemia and plasma lactate

\begin{tabular}{llll}
\hline & \multicolumn{3}{l}{ Lactate, $\mathbf{m m o l} / \mathbf{L}(\mathbf{n}=159)$} \\
\cline { 2 - 4 } & $\mathbf{r}$ & $\boldsymbol{\beta}$ & $\mathbf{P}$ value* \\
\hline $\begin{array}{l}\text { Serum fasting } \\
\text { glucose, } \mathrm{mg} / \mathrm{dL}\end{array}$ & 0.19 & 0.008 & 0.01 \\
$\begin{array}{l}\text { Serum 2-hour } \\
\text { glucose, } \mathrm{mg} / \mathrm{dL}\end{array}$ & 0.19 & 0.003 & 0.005 \\
$\begin{array}{l}\text { Serum fasting } \\
\text { insulin, } \mu \mathrm{U} / \mathrm{mL}\end{array}$ & 0.33 & 0.03 & $<0.001$ \\
$\begin{array}{l}\text { Serum 2-hour } \\
\text { insulin, } \mu \mathrm{U} / \mathrm{mL}\end{array}$ & 0.18 & 0.002 & 0.02 \\
$\begin{array}{l}\text { Homeostasis model } \\
\text { assessment, units }\end{array}$ & 0.33 & 0.11 & $<0.001$ \\
\hline
\end{tabular}

${ }^{*} P$ value for ordinary least squares coefficient ( $\beta$ ) adjusted for age, sex, and race/ethnicity.

$\beta$, beta coefficient; $r$, Pearson's correlation coefficient.

reduction in the lactate level of $0.08 \mathrm{mmol} / \mathrm{L}$ (compared with a high GI, high carbohydrate proportion diet). Thus, the effects of GI and carbohydrate were not additive. These effects did not differ by demographic or clinical characteristics. Moreover, lactate was significantly associated with markers of insulin resistance, including serum fasting glucose, serum 2-hour glucose, serum fasting insulin, serum 2-hour insulin, and HOMA. These findings show that in adults at risk for diabetes due to obesity, reducing the GI and/or the amount of dietary carbohydrates affects short-term metabolism.

Our study demonstrated that reducing GI and reducing total carbohydrates lowered plasma lactate concentrations, a marker of non-oxidative glycolysis. Lactic acid (2-hydroxypropanoic acid) is a downstream product of glycolysis that forms when pyruvate is diverted from the Krebs cycle. ${ }^{14}$ Lactate production increases when energy demand exceeds mitochondrial oxidative capacity. High lactate levels can also arise in settings of excess intracellular glucose such as in von Gierke's disease. ${ }^{2526}$ There is some evidence to suggest that lactate is a marker of glucose stores in peripheral tissue that can reduce insulin sensitivity in the long term. ${ }^{27}$

Lactate is a possible predictor of diabetes mellitus, independent of glucose and insulin. ${ }^{12}$ Furthermore, lactate's association with diabetes has been observed in large population studies, cross-sectionally and prospectively. ${ }^{11} 1{ }^{1316}$ In the Atherosclerosis Risk in Communities cohort study, the association between lactate and incident diabetes was prominent in the subpopulation with pre-diabetes, suggesting a role of lactate as a predictor of diabetes in an at-risk population. ${ }^{12}$ In persons without diabetes, basal lactate level was found to be negatively associated with insulin sensitivity, even after adjustment for obesity. ${ }^{16}$ Speculation of mechanisms of the association between lactate and incident diabetes is beyond the scope of our present study. 
We showed that lowering the amount of carbohydrates in the setting of high GI lowered lactate. A similar observation has been reported in other studies. In one trial of 19 subjects, a high carbohydrate diet, compared with diets high in protein, fat, or alcohol, led to higher postprandial lactate. ${ }^{28}$ In another study of eight subjects undergoing exercise, a low carbohydrate diet, compared with a regular carbohydrate diet, led to delayed production of lactate after exercise. ${ }^{29}$ It has been hypothesized that reduced lactate levels may be due to decreased glycogen levels or decreased flux through glycogenolysis or anaerobic glycolysis. ${ }^{30}$ Alternatively, the shift in substrate for oxidative phosphorylation resulting from reduced carbohydrate availability may mean that a greater portion of acetyl coenzyme A may be derived from fat and protein resources, thereby bypassing glycolysis.

Our study also showed that lowering GI in the context of high carbohydrates non-significantly reduced lactate. In a study of 12 subjects and another of 19 subjects, carbohydrate type (sucrose, which is a simple carbohydrate, vs starch, which is a complex carbohydrate) did not affect blood lactate levels. ${ }^{31}{ }^{32}$ Of note, these trials did not distinguish carbohydrates by GI, as they were conducted before classification of carbohydrates by GI was widely adopted. More recently, a study of eight subjects showed that low GI diet, compared with high GI diet, led to a smaller rise in plasma lactate after exercise. ${ }^{33}$ The longterm implications of these changes should be the focus of subsequent research.

Our study has limitations. First, the feeding periods were too brief for observation of clinical events, such as incident diabetes mellitus, thus necessitating examination of surrogate markers as outcomes. Second, the high carbohydrate diet was compared with a low carbohydrate diet that had simultaneous increases in both protein and fat. The effects of increasing protein and fat cannot be distinguished from lowering carbohydrates. The intervention was not designed to examine subtypes of protein or fat (eg, animal vs plant-based protein or unsaturated vs saturated fat). Similarly, while the diets were designed to have similar fiber levels between diets, it is possible that whole grains or specific ingredients in each diet contribute to changes in lactate levels. While we attempted to maintain fiber content constant across diets, there was slightly more fiber in the high carbohydrate, low GI diet. Nevertheless, the comparison of the lowest versus the highest fiber diets (discordant contrast: cG vs $\mathrm{Cg}$ ) did not significantly affect lactate. Adding wheat bran as a source of fiber could alter the GI of some foods. This should be noted when attempting to replicate the meals prepared in this trial. Last, all diets reduced plasma lactate level. However, our study cannot distinguish between whether these changes from baseline reflect the benefits of a supervised dietary intervention or low-quality diets at home.

Our study also has multiple strengths. First, the trial was a randomized, controlled feeding study with high adherence and large contrasts in the dietary exposures examined in OmniCarb (GI and \% kcal carb) ${ }^{7}$ Second, the feeding periods were designed to be isocaloric, allowing us to isolate the dietary factors without the confounding effects of weight change. Third, plasma lactate and other biomarkers were measured over multiple standardized blood collections in a population without diabetes or glucose-altering medications. Fourth, the study population was diverse in sex and race, with few participants lost to follow-up. Finally, OmniCarb was a cross-over trial, reducing the influence of confounding covariates.

This study has implications for our understanding of the role of carbohydrates in adults at risk for diabetes. There is ongoing debate regarding whether a high GI, high carbohydrate diet contributes to diabetes. ${ }^{334}$ Some argue that carbohydrates are only relevant for glucose management in patients with established diabetes, and that they do not have a role in the development of diabetes independent of weight and obesity. ${ }^{6}$ While our study did not show effects of carbohydrates on shortterm glucose homeostasis, we did show that reducing either GI or amount of carbohydrates lowered plasma lactate, a measure of both intracellular metabolism and diabetes risk. Future research is needed to determine whether this cellular shift toward more efficient metabolism affects body weight, an important causal mediator of diabetes.

In conclusion, we found that reducing the GI and amount of dietary carbohydrates affected carbohydrate metabolism reflected by lower lactate concentrations. These findings were observed in a population at risk for diabetes despite minimal effects on glucose and insulin. While lactate has been implicated in diabetes pathogenesis in other studies, further research is needed to determine whether the metabolic changes we observed in OmniCarb decrease diabetes risk in the long term.

\section{Author affiliations}

${ }^{1}$ Department of Medicine, Vanderbilt University Medical Center, Nashville, Tennessee, USA

${ }^{2}$ Welch Center for Epidemiology, Prevention, and Clinical Research, Johns Hopkins University Bloomberg School of Public Health, Baltimore, Maryland, USA

${ }^{3}$ Department of Nutrition, Harvard University T H Chan School of Public Health, Boston, Massachusetts, USA

${ }^{4}$ Division of General Medicine and Primary Care, Beth Israel Deaconess Medical Center, Boston, Massachusetts, USA

Twitter Jiun-Ruey Hu @ruey_hu, Lawrence J Appel @LarryAppel, Edgar R Miller III @ermiller14 and Stephen P Juraschek @spjuraschek

Contributors SPJ designed the study, conducted data collection, performed primary data analyses, and critically reviewed the manuscript. J-RH interpreted the data and wrote the paper. YW performed secondary data analysis. FMS, LJA, and JHY provided critical reading of the manuscript and comments. ERM designed the study and provided critical reading of the manuscript and comments. All coauthors provided final approval of the article. SPJ takes final responsibility for the final content.

Funding The OmniCarb study was supported by NIH grants HL-084568 and HL-084568. SPJ's work was supported by K23HL135273 and R21HL144876. J-RH was supported by the Linda Kao Memorial Fund.

Competing interests None declared. 
Patient consent for publication Not required.

Ethics approval The study protocol was approved by the institutional review boards at Johns Hopkins University (IRB\# NA_00014138) and the Harvard School of Public Health (IRB\# 2007 P-001351).

Provenance and peer review Not commissioned; externally peer reviewed.

Data availability statement Data are available upon reasonable request.

Open access This is an open access article distributed in accordance with the Creative Commons Attribution Non Commercial (CC BY-NC 4.0) license, which permits others to distribute, remix, adapt, build upon this work non-commercially, and license their derivative works on different terms, provided the original work is properly cited, appropriate credit is given, any changes made indicated, and the use is non-commercial. See: http://creativecommons.org/licenses/by-nc/4.0/.

ORCID IDs

Jiun-Ruey Hu http://orcid.org/0000-0003-1390-508X

Stephen P Juraschek http://orcid.org/0000-0003-4168-2696

\section{REFERENCES}

1 Centers for Disease Control and Prevention. National diabetes statistics report, 2017. Atlanta, GA: Centers for Disease Control and Prevention, 2017.

2 Sainsbury E, Kizirian NV, Partridge SR, et al. Effect of dietary carbohydrate restriction on glycemic control in adults with diabetes: a systematic review and meta-analysis. Diabetes Res Clin Pract 2018;139:239-52.

3 Thomas D, Elliott EJ, Cochrane Metabolic and Endocrine Disorders Group. Low glycaemic index, or low glycaemic load, diets for diabetes mellitus. Cochrane Database Syst Rev 2009;14.

4 Jenkins DJ, Wolever TM, Taylor RH, et al. Glycemic index of foods: a physiological basis for carbohydrate exchange. Am J Clin Nutr 1981:34:362-6.

5 Brand-Miller JC, Astrup A, Buyken AE. Low vs high glycemic index diet. JAMA 2015;313:1371-2.

6 Eckel RH. Role of glycemic index in the context of an overall hearthealthy diet. JAMA 2014;312:2508-9.

7 Sacks FM, Carey VJ, Anderson CAM, et al. Effects of high vs low glycemic index of dietary carbohydrate on cardiovascular disease risk factors and insulin sensitivity: the OmniCarb randomized clinical trial. JAMA 2014;312:2531-41.

8 Juraschek SP, Miller ER, Selvin E, et al. Effect of type and amount of dietary carbohydrate on biomarkers of glucose homeostasis and $C$ reactive protein in overweight or obese adults: results from the OmniCarb trial. BMJ Open Diabetes Res Care 2016;4:e000276.

9 Juraschek SP, Miller ER, Appel LJ, et al. Effects of dietary carbohydrate on 1,5-anhydroglucitol in a population without diabetes: results from the OmniCarb trial. Diabet Med 2017;34:1407-13

10 Crawford SO, Hoogeveen RC, Brancati FL, et al. Association of blood lactate with type 2 diabetes: the Atherosclerosis risk in communities carotid MRI study. Int J Epidemiol 2010;39:1647-55.

11 Juraschek SP, Shantha GPS, Chu AY, et al. Lactate and risk of incident diabetes in a case-cohort of the Atherosclerosis risk in communities (ARIC) study. PLoS One 2013;8:e55113.

12 Juraschek SP, Selvin E, Miller ER, et al. Plasma lactate and diabetes risk in 8045 participants of the Atherosclerosis risk in Communities study. Ann Epidemiol 2013;23:791-6.

13 Ohlson LO, Larsson B, Svärdsudd K, et al. The influence of body fat distribution on the incidence of diabetes mellitus. 13.5 years of follow-up of the participants in the study of men born in 1913. Diabetes 1985;34:1055-8.

14 Adeva-Andany M, López-Ojén M, Funcasta-Calderón R, et al. Comprehensive review on lactate metabolism in human health. Mitochondrion 2014;17:76-100.
15 Del Prato S, Bonadonna RC, Bonora E, et al. Characterization of cellular defects of insulin action in type 2 (non-insulin-dependent) diabetes mellitus. J Clin Invest 1993;91:484-94.

16 Lovejoy J, Newby FD, Gebhart SS, et al. Insulin resistance in obesity is associated with elevated basal lactate levels and diminished lactate appearance following intravenous glucose and insulin. Metabolism 1992;41:22-7.

17 Lovejoy J, Mellen B, Digirolamo M. Lactate generation following glucose ingestion: relation to obesity, carbohydrate tolerance and insulin sensitivity. Int J Obes 1990;14:843-55.

18 Miller BF, Fattor JA, Jacobs KA, et al. Lactate and glucose interactions during rest and exercise in men: effect of exogenous lactate infusion. $J$ Physiol 2002;544:963-75.

19 Lombardi AM, Fabris R, Bassetto F, et al. Hyperlactatemia reduces muscle glucose uptake and GLUT-4 mRNA while increasing (E1 $\alpha)$ PDH gene expression in rat. Am J Physiol Endocrinol Metab 1999;276:E922-9.

20 Ahlborg G, Felig P. Lactate and glucose exchange across the forearm, legs, and splanchnic bed during and after prolonged leg exercise. J Clin Invest 1982;69:45-54.

21 DiGirolamo M, Newby FD, Lovejoy J. Lactate production in adipose tissue: a regulated function with extra-adipose implications. Faseb $J$ 1992:6:2405-12.

22 Michaud DS, Fuchs CS, Liu S, et al. Dietary glycemic load, carbohydrate, sugar, and colorectal cancer risk in men and women. Cancer Epidemiol Biomarkers Prev 2005;14:138-47.

23 National Cancer Institute. Diet history questionnaire II (DHQ II) forms | EGRP/DCCPS/NCI/NIH, 2010. Available: https://epi.grants.cancer. gov/dhq2/forms/ [Accessed 9 Jun 2020].

24 Matthews DR, Hosker JP, Rudenski AS, et al. Homeostasis model assessment: insulin resistance and beta-cell function from fasting plasma glucose and insulin concentrations in man. Diabetologia 1985;28:412-9.

25 Jones JG, Garcia P, Barosa C, et al. Hepatic anaplerotic outflow fluxes are redirected from gluconeogenesis to lactate synthesis in patients with type 1a glycogen storage disease. Metab Eng 2009;11:155-62.

26 Oster Y, Wexler ID, Heyman SN, et al. Recoverable, Record-High lactic acidosis in a patient with glycogen storage disease type 1: a mixed type A and type B lactate disorder. Case Rep Med 2016;2016:4362743.

27 McDermott JC, Bonen A. Glyconeogenic and oxidative lactate utilization in skeletal muscle. Can J Physiol Pharmacol 1992;70:142-9.

28 Raben A, Agerholm-Larsen L, Flint A, et al. Meals with similar energy densities but rich in protein, fat, carbohydrate, or alcohol have different effects on energy expenditure and substrate metabolism but not on appetite and energy intake. Am J Clin Nutr 2003;77:91-100.

29 Langfort J, Czarnowski D, Zendzian-Piotrowska M, et al. Short-term low-carbohydrate diet dissociates lactate and ammonia thresholds in men. J Strength Cond Res 2004;18:260-5.

30 Jacobs I. Lactate concentrations after short, maximal exercise at various glycogen levels. Acta Physiol Scand 1981;111:465-9.

31 Kelsay JL, Behall KM, Moser PB, et al. The effect of kind of carbohydrate in the diet and use of oral contraceptives on metabolism of young women. I. blood and urinary lactate, uric acid, and phosphorus. Am J Clin Nutr 1977;30:2016-22

32 Solyst JT, Michaelis OE, Reiser S, et al. Effect of dietary sucrose in humans on blood uric acid, phosphorus, fructose, and lactic acid responses to a sucrose load. Nutr Metab 1980;24:182-8.

33 Thomas DE, Brotherhood JR, Brand JC. Carbohydrate feeding before exercise: effect of glycemic index. Int J Sports Med 1991;12:180-6.

34 Sheard NF, Clark NG, Brand-Miller JC, et al. Dietary carbohydrate (amount and type) in the prevention and management of diabetes: a statement by the American diabetes association. Diabetes Care 2004;27:2266-71. 